\title{
Motor neurone disease: towards better care
}

\author{
FORBES H NORRIS, RICHARD A SMITH, ERIC H DENYS
}

\begin{abstract}
Even in the most severe forms of motor neurone diseaseprogressive bulbar palsy and amyotrophic lateral sclerosis-the symptoms and disabilities from progressive paralysis may be relieved in many patients by various symptomatic treatments. Quality of life may be improved even in the terminal stage, when narcotic administration should be considered. The physician's proper role is to offer and carefully supervise these treatments, not withhold them. Home care is recommended even for the most severely paralysed, though hospice care may be a good alternative. The underlying principle-to alleviate symptoms-applies to the management of all progressive incurable diseases.
\end{abstract}

\section{Introduction}

Motor neurone disorders, particularly the most malignant-progressive bulbar palsy and amyotrophic lateral sclerosis-provide an astonishing example of therapeutic ignorance or nihilism in modern medical practice. ${ }^{1}$ While some types of motor neurone disease are relatively benign, the most common is amyotrophic lateral sclerosis, which in its progression exacts a very severe toll, not only on the sufferer but usually on the family as well. Some cases of progressive bulbar palsy remain relatively limited, but even so the illness is usually as devastating. Many medical attendants are so appalled that they do not think "it right to embark on measures likely to prolong distress ... [such as] gastrostomy or tracheostomy."

These patients may, however, live many months and even many years, so withholding supportive and symptomatic treatments adds appreciably to the duration of suffering. On the other hand, providing such treatment often increases the sufferer's quality of life in the remaining time. It has been a decade since we recommended a course of various symptomatic treatments for amyotrophic lateral sclerosis, ${ }^{2}$ so it seems timely to provide an update, including more details in some aspects as well as a look at newer treatment options. To this problem we bring personal experience in the daily care of over 1000 patients with motor neurone disorders of all types.

\section{Sleep disorders}

Sleep disorders are very common in paralysed patients, perhaps almost universal at one time or another, probably because paralysis prevents the physical activities that somehow promote sleep. If sleep occurs naturally at

\footnotetext{
ALS and Neuromuscular Research Center and Department of Neurology, Pacific Presbyterian Medical Center, San Francisco, California 94120 FORBES H NORRIS, MD

ERIC H DENYS, MD

Center for Neurologic Study and Scripps Hospital, San Diego, California 92121 RICHARD A SMITH, MD

Correspondence to: Dr F H Norris.
}

the customary hour it may be appreciably shortened on this account, and also by any complicating psychological problem such as depression. Many patients and their families have described short naps during the day in addition to any sleep during the night, but this does not appear to satisfy in most cases. Probably a contributing factor is the myth inculcated since childhood that fixed hours of sleep each night are essential to preserve health. Sleep apnoea may also be a factor.

Rather than supply a sedative automatically we prefer to open this aspect of treatment by discussing the problem with the patient and family in general terms, in search of other factors, amelioration of which may improve sleep. For example, many patients with amyotrophic lateral sclerosis suffer from painful cramps and in some these are nocturnal; suppression of the cramps by appropriate medication (see below) would be an obvious first step. Many of these patients experience minor aches and pains during the night from inability to move normally, and the cumulative effect may be interruption of sleep. Some patients with such a secondary sleep disorder obtain relief by using an electrically powered bed. Most of these patients have the strength to push a buttom to raise the knees, and this simple change may substitute for natural movements during sleep.

Patients with motor neurone disorders tend to be an older group, ${ }^{1}$ and if a sleeping medication is required care must be taken to ensure that it is well tolerated. A particularly useful agent is diphenhydramine (see table II), which suppresses cramps and also promotes sleep very safely. Chloral hydrate is helpful as a sedative in many patients.

Care must also be taken to ensure that patients who have or are likely to develop respiratory failure are not prescribed a sedative having a particularly depressant effect on respiration. Potentially all of the benzodiazepines may depress respiration, and we find that this is particularly true of diazepam. Flurazepam (15-45 mg), however, is comparatively safe as a sleeping medication for many patients. The doses of all these drugs should be minimised, and often the patient's understanding of the problem will lead to better tolerance of decreased hours of sleep, hence less call for medication.

\section{Constipation}

Probably also owing to lack of the usual daily physical activities, constipation becomes an increasing problem in amyotrophic lateral sclerosis. ${ }^{3}$ As in sleep disorders, careful discussion with the patient and the family often gives understanding which increases tolerance of the problem (most people of Western cultures are convinced that regular bowel evacuations are not only healthy but that irregularity or delay is positively harmful). A careful aperient history is essential, as clues may be obtained about substances useful in the past. Most people have noted at least mild cathartic effects from caffeine and citrus fruits. Perhaps the old saying "An apple a day keeps the doctor away" originated in appreciation of the cathartic effect of apple pectin. If such simple (and nutritious) remedies have not been tried, then this is the obvious first step.

Since bulbar palsy often causes most swallowing difficulty with clear liquids, which "fall apart" in swallowing and thus lead to aspiration, these patients often avoid water and other clear liquids and thus increase constipation by dehydration. In such cases, watery soft foods-for example, sherbets, custards, apple sauce, etc-may bring considerable relief. Even normal meals can be blenderised with water, fruit juices, diluted wine, and other liquids to produce palatable but less dysphagic food preparations (see below).

Another approach to severe constipation, including faecal impaction, is review of the other medications in a particular patient. All anticramp and antispasm medications have potential for causing or increasing constipation, as do sedatives. Among the medications useful to reduce drooling (see below), glycopyrronium, methanthelinium, and propantheline may also reduce bowel motility. 
If these simple measures fail then use of senna extracts may be helpful, in widely varying dosages, which may be mixed with an equal amount of Milk of Magnesia. Intermittent use of packaged phosphate enemas may be prescribed. Manual disimpaction ${ }^{1}$ should be a rare event. If it becomes necessary we prefer to send a visiting nurse to the home or bring the patient into the emergency room rather than permit disimpaction by the spouse, ${ }^{1}$ which is destructive of both partners' morale.

\section{Dysphagia}

Impaired swallowing ability usually includes reduction of reflex glottic closure, so that dysphagia and aspiration occur concomitantly. Aspiration may be manifested only by a dry cough increased at mealtime. At least early in such bulbar disorder, judicious use of blenderised food and avoidance of strong spices and irritating food and drink are by far the best ways to approach this problem. The physician can help by prescribing medications in liquid form or by advising about pulverising suitable medications.

Ordinarily bizarre food and drink combinations may be blenderised to achieve both palatability and less dysphagia. Examples include ground beef with oranges and cranberries, diluted with either fruit juice plus red wine or water; scrambled eggs, bacon, and pancakes diluted with orange or cranberry juice plus water or coffee; and broiled or roasted chicken with broccoli, lemon juice, and white wine or water.

One cause of upper oesophageal obstruction and dysphagia in mainly spastic patients is painless cricopharyngeal muscle spasm, ${ }^{4}$ which we find is not relieved by anticramp medications. Our early experience with cricopharyngeal myotomy included frequent treatment failures and major complications (usually pulmonary after the general anaesthesia). ${ }^{5}$ We now select patients more rigorously, ${ }^{6}$ using the following criteria: dysphagia including a globus sensation; recent weight loss exceeding $15 \%$ of body weight; relative preservation of lower motor neurone bulbar functions but severe impairment of upper motor functions and a hyperactive jaw jerk; forced vital capacity greater than $50 \%$ of predicted and maximum voluntary ventilation greater than $25 \%$ of predicted. ? The incidences of major morbidity and treatment failure now total less than $5 \%$. In successful cases about $15 \%$ of patients are also able to speak more clearly. As in the reduction of oropharyngeal secretions (see below) we surmise that such improvement results from reduction of hyperactive pharyngeal-laryngeal reflex systems.

Since all types of motor neurone disease are usually progressive most patients develop more severe dysphagia and secondary malnutrition requiring bypass of the oropharyngeal mechanism. In some cases rapidly progressive weight loss is associated with accelerating weakness. Both the weight loss and the additional weakness have sometimes been stopped-and occasionally reversed to some degree-by high energy tube feeding. A nasogastric tube is the simplest approach.

For some years we recommended an oesophagostomy in preference to classical gastrostomy or jejunostomy if the nasogastric tube was not tolerated. A cervical oesophagostomy may be performed easily and safely with the patient under local or general anaesthesia. Within a month a cutaneoesophageal fistula develops and the tube can then be changed easily. Patients adjust quickly to a cervical oesophagostomy. A shirt collar, handkerchief, or ascot disguises its presence. It was difficult to find a surgeon skilled in the procedure and willing to perform it under local anaesthesia; general anaesthesia at this stage often leads to pulmonary complications.

Recently application of percutaneous catheter gastrostomy ${ }^{8}$ has been a simple, safe, effective procedure for our malnourished, dysphagic patients, especially when severe pulmonary muscle weakness greatly increased the risk from anaesthesia. ${ }^{9}$

We disagree strongly with those who "have not thought it right to embark on measures likely to prolong distress, and no patients were submitted to cricopharyngeal myotomy, gastrostomy, or tracheostomy and only one patient was fed by a nasogastric tube"1-in reference to all patients, presumably including some in non-terminal stages. Is the amelioration of constant gagging and choking not a reasonable therapeutic outcome? Is adequate hydration likely to prolong life more than a few days or weeks? Is it preferable to die of starvation and thirst, as well as amyotrophic lateral sclerosis, because the attending doctors do not think "it right" to relieve the additional suffering?

\section{Excessive secretions}

Table I lists medications useful in the suppression of drooling and the reduction of oropharyngeal secretions, accumulation of which promotes aspiration. Sometimes a reduction of the secretions improves speech, perhaps by modulating the pharyngeal and laryngeal reflexes. The particular medication of most benefit in a given case is unpredictable; we prefer to start with the safer, more economical ones such as methanthelinium and $\square$ propantheline, then progress to the others if this first effort fails. Some patients get good relief from patent "decongestants" available for relief of colds and hay fever. We have seen many patients whose other physicians $\varrho$ prescribed atropine for this secretion problem. In older patients atropine $\subsetneq$

TABLE I-Medications useful in suppressing excessive oropharyngeal secretions and drooling

\begin{tabular}{llll}
\hline Medication & Dosage and schedule & Medication & Dosage and schedule \\
\hline Methanthelinium & $50-100 \mathrm{mg}$ four hourly & Methylphenidate & $10-20 \mathrm{mg}$ six hourly \\
Propantheline & $15-30 \mathrm{mg}$ four hourly & Clonidine & $0.1 \mathrm{mg}$ six hourly \\
Glycopyrronium & $1-2 \mathrm{mg}$ four hourly & Benzhexol & $2-10 \mathrm{mg}$ four hourly \\
Amitriptyline & $10-50 \mathrm{mg}$ four hourly & Hyoscine & Transdermal application \\
Imipramine & $50-200 \mathrm{mg}$ at bedtime & & \\
\hline
\end{tabular}

^Usual adult dosage.

sometimes causes paranoia or hallucinations, and there are the usual $\vec{\circ}$

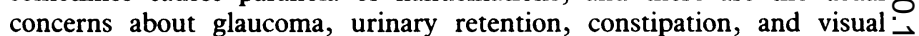
blurring. Recently some patients have seemed to obtain good results from $\vec{\omega}$ skin applications of hyoscine (TransDerm). There remain treatment $\mathscr{N}$ failures, and particularly a few patients in whom the medications convert the $\frac{5}{7}$ secretions into thick, tenacious strings which are even more difficult to manage.

\section{Cramps and spasms}

Table II shows the medications useful for cramps. Diphenhydramine is mentioned above as being particularly useful at bedtime. Quinine, in $ᄋ$ effective dosage, often produces tinnitus and itching, and sometimes $N$ vertigo, but some patients obtain a good result with the doses listed. The use of quinidine and procainamide needs careful consideration in an older $\subseteq$ patient or a patient of any age with heart disease. Baclofen is extremely helpful in most cases, the main problems being lack of any effect at the $\vec{\varphi}$ recommended dosage (up to $80 \mathrm{mg} /$ day) and complaints of lethargy and $\stackrel{O}{\circ}$ mental slowing (fewer than $2 \%$ of patients). These side effects are $\square$ encountered more frequently with diazepam. Dantrolene is available for trial but seems to be poorly tolerated by this group of patients. ${ }^{10}$

TABLE II-Medications useful in relieving spastic symptoms

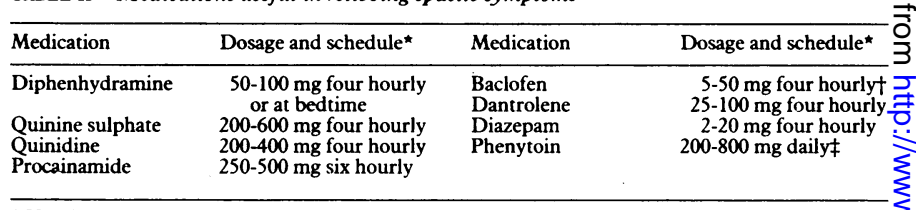

^ Usual adult dosage. dosages (up to $80 \mathrm{mg} /$ day) but obtain appreciable relief from higher doses.

$\ddagger$ Phenytoin for this purpose seems to be potentiated by addition of calcium gluconate $0.5-1.0 \mathrm{~g}$ every four to six hours.

Simpler, safer, and more rapidly effective is the manoeuvre of stretching $N$ the cramped muscle. ${ }^{11}$ Occasionally both agonist and antagonist muscles will be severely cramped and another person's help is needed, or the cramps may affect the thoracic or abdominal muscles, where stretching is difficult; or the sufferer may be alone and too weak to stretch the muscle, so in these cases $N$ medication is preferable.

All of the medications for cramp may be tried for the reduction of 0 spasticity, in particular leg scissoring due to spasm of adductor muscles. The results are not as good in ambulatory patients because the necessary dosage may reduce the "spastic crutch" which provides some gait stability.

\section{Fatigue}

Fatigue may be due to reactive depression and respond to such treatment. Neuromuscular fatigue sometimes responds to a trial of an anticholinesterase, ${ }^{12}$ but the response correlates poorly with electrographic tests of $O$ neuromuscular transmission in amyotrophic lateral sclerosis because individual muscles have varying degrees of synaptic failure. ${ }^{13} \mathrm{We}$ usually begin with an empirical trial of pyridostigmine $30 \mathrm{mg}$ with each meal, increasing over several weeks to no more than $120 \mathrm{mg}$ with meals. Neostigmine and choline theophyllinate are also available for trial. 


\section{Exercise}

We have observed repeatedly that all patients with motor neurone disorders seem unusually susceptible to disuse atrophy and weakness. Patients who become despondent and stay in bed seem to lose ground more rapidly than before, and also acquire further disability from contractures. In a small group with relatively benign illness (slower courses, long intervals of stability, etc) a common denominator is daily active exercises. ${ }^{14}$ Lenman showed in a scientific study that active exercise is helpful. ${ }^{15}$ Remarkable improvement from daily swimming has been documented in a patient with slowly progressive lower motor neurone disease. ${ }^{16}$

Although active exercises for patients with amyotrophic lateral sclerosis are not usually prescribed by most physicians, we believe that they are indicated, particularly when the physical activity is enjoyable and not exhausting. When there is noticeable leg weakness (so falling is a risk) exercise in a heated pool should be recommended. Problems are finding a pool which is open in the winter and provides the disabled with a mechanical lift for exit from the water. We have seen two patients with amyotrophic lateral sclerosis who became transiently weaker when too warm, but most reported transient weakness with chilling. This is the opposite of common experience in multiple sclerosis. But some of our patients have never been in a pool and use daily gymnastic exercises, others cycle, and so on.

Passive exercises should be directed at the weakest parts in order to maintain joint mobility and muscle tone. The patient and family or friends can manage most exercise programmes at home. The shoulder should receive periodical professional scrutiny in all cases of amyotrophic lateral sclerosis because of the high risk of subluxation with resulting painful periarthritis, and contractures (frozen shoulder) increasing the original disability. We suggest a combined active-passive exercise programme organised or modified by professionals whenever the patient with amyotrophic lateral sclerosis is in hospital, with monthly to quarterly revisions at home. As with home respirator care, motivated family members and even friends can be trained rapidly to administer physiotherapy several times daily, ${ }^{17}$ which is far more important than brief professional sessions once or twice a week.

\section{Aids}

We have received many protests from insurance carriers that aids such as splints, corrective braces, grab bars, raised toilet seats, reach devices, and so on $^{18}$ cannot be provided because bulbar palsy and amyotrophic lateral sclerosis are progressive and fatal and such devices do not prevent their outcome. This attitude helps reduce any hope the sufferer may have summoned and accelerates the original self defeating prophecy. ${ }^{1}$ As we and others have shown elsewhere, amyotrophic lateral sclerosis is not invariably progressive: short plateaus are common, relatively benign courses occur in $20 \%$ of patients, and "burn out" may occur in a smaller minority. Periods of increased function, usually to small degree, may occur during any of the stable periods. So refusal of these devices on the assumption of further, rapid progression in the near future is not justified, and any such official transmission of the refusal may cause psychological deterioration in a given patient. Who can say whether they will help for three weeks or three years? Who can say, even though they may have helped for only three weeks, that it was not a useful expenditure for that patient?

\section{Communication}

We regret passing over the major problem of communication in severe bulbar disease, ${ }^{19}$ but so many advances are being reported that it seems likely that anything described today will be outdated within a year; moreover, this topic really deserves separate, lengthy treatment. Microcomputer systems provide anarthric patients with the ability to communicate their needs, though no system rivals natural speech. Probably for anarthric patients with some finger strength the single most useful device so far is the Canon "Communicator." 20

\section{Respiratory insufficiency}

Respiratory insufficiency develops sooner or later in most cases of amyotrophic lateral sclerosis. We have seen, and others have reported, patients in whom relatively acute respiratory insufficiency heralded the onset of the disease, but usually this life threatening paralysis appears gradually as the other symptoms emerge and intensify. The earliest nocturnal symptoms usually occur because the patient is sleeping horizontally and the viscera compromise the diaphragm. When the head of the bed is raised $30-40^{\circ}$ gravity decompresses the diaphragm and relieves the symptom.
Some patients obtain so much benefit that, as the illness progresses, they use more and more elevation, eventually reaching the erect position. For these patients we recommend purchase of a reclining lounge chair with leg rest.

At this stage in the illness more severe respiratory problems obviously loom, and the observant physician's duty is to alert the patient and the family. Bradley has well described this situation and the neurologist's role. ${ }^{21} 22$ One important consideration is the relative benefit (versus respiratory depressant side effects) of any current medications to relieve or prevent cramps and spastic symptoms (table II). If, for example, the cramp problem is mild but the cramp medication is diazepam $20 \mathrm{mg}$ every four hours, it is desirable to reduce the dosage or even stop that drug to ascertain any part that it may be playing in the respiratory failure. As noted above, all the anticramp medications may depress respiration in sensitive patients and in most patients receiving high dosages.

If the respiratory symptoms fail to respond to simple measures or worsen then a fatal outcome is likely unless ventilatory support is provided. ${ }^{23}{ }^{24}$ Some of our patients get temporary relief from use of a rocking bed at night, cuirass respirators, or a "pneumobelt" to provide periodic visceral (and secondary thoracic) compression. If intubation is necessary then tracheostomy is the next decision, and this should always be the patient's option. ${ }^{25} \mathrm{~A}$ drastic, unexpected change in pulmonary function may mandate emergency and involuntary intubation with use of artificial ventilation, but there is time subsequently to review the entire position and to fully inform the patient about the options.

If the patient in critical respiratory failure opts against intubation and artificial ventilation, and does so rationally and forcefully, the physician has an obligation to honour this direction ${ }^{25}$; and at this point we offer such patients morphine or pethidine to relieve any subsequent suffering. If the patient opts for artificial respiration then tracheostomy is performed electively. Our recent experience shows that $90 \%$ of these patients may then go home, so the prospect of lifelong stay in the intensive care unit need not enter the consideration about going on a life support system. ${ }^{17}$

\section{Unorthodox treatment}

Most patients will wish to take vitamins, and many will seek unorthodox treatment. It is probably wise to acquiesce, at least when the proposed treatment is not obviously risky or inordinately expensive. The physician should not interpret these ventures as loss of confidence in his ministrations, nor reject the patient who seeks unorthodox help.

Acupuncture has had an appeal in the past decade. Hundreds of our patients have tried it without noticeable benefit, although four became jaundiced afterwards, presumably from contaminated needles.

An important factor in every illness, particularly in progressive paralytic diseases like amyotrophic lateral sclerosis, is patient morale. Often the self trial of an unorthodox treatment aids morale because the patient is trying something that he has found. In the era of heart transplantation it is probably asking too much for patients with amyotrophic lateral sclerosis to rely completely on their physicians, who do not even know the cause of the disease.

\section{Emotional lability}

One of the most demoralising problems in upper motor bulbar palsy is loss of emotional control, which may affect the patient, family, friends, and medical attendants. Everyone is demoralised by sustained weeping or laughter, or laughter making a transition to frustrated weeping. In a small series we observed appreciable amelioration from administration of lithium carbonate $300 \mathrm{mg}$ given once to three times a day. Sporadic serum lithium concentrations were always less than $0.6 \mathrm{mmol}(\mathrm{mEq}) / 1$, so this effect was not likely to include the neural pathways affected by lithium in manic depressive psychosis. Levodopa may also be of benefit. ${ }^{26}$

\section{Terminal state}

Unless tracheostomy and artificial ventilation are elected, the dying patient lapses into a light coma. The main concern at this stage is the patient's comfort. The family should be in attendance. If feasible, the entire process should occur at home, among familiar objects and faces. The hospice has been of great help in some cases in recent years, particularly when the spouse must work outside the home or cannot cope.

New or inconsistent symptoms such as bloody sputum or swollen legs should be neither investigated nor treated vigorously. It is immaterial, and therefore should not be tested, whether the serum sodium concentration is 129 or $139 \mathrm{mmol}(\mathrm{mEq}) / 1$. The physician's last useful act for the patient should be a generous prescription of narcotics. ${ }^{3}$ The risk of addiction does 
not exist and dose increases should be ordered routinely to ensure the patient's comfort in the face of distress from aspiration, cramps, joint pains, and so forth. Larger than standard narcotic dosages are often necessary right away because most of the patients will have been receiving the minor narcotics already for the various painful symptoms caused by the illness (see above). Certainly if there is any other painful concurrent condition its treatment should be continued as well. At this stage parenteral or tube administration of medications should be employed.

We usually place an indwelling bladder catheter to ensure a dry bed. Again the risk of urinary tract infection is less important than the patient's immediate comfort.

\section{Concluding remarks}

Modern medical professionals are strongly orientated towards specific and particularly curative treatments, ranging most dramatically from heart transplantation to more humble physiotherapy. This orientation comes not just from the natural human inclination to focus on solvable problems and from the teaching orientation in the schools but also from outside influences, such as government requirements that rehabilitation services should be provided only where recovery can be expected. In the United States convalescent care is limited to a specific number of days per lifetime, as though chronic illness of longer duration were non-existent. These are relatively new developments, and probably physicians earlier in this century were much more attuned to the possibilities of successful care through the administration of symptomatic treatments.

Our example is the application of specific symptomatic treatments to the care of patients suffering from the motor neurone diseases, particularly amyotrophic lateral sclerosis. We emphasise the administration of treatments at home because of the importance of the home in the maintenance of morale, although a secondary gain from home treatment is a noticeable reduction in the cost of care.

Our earlier publication on this topic ${ }^{2}$ was directed at principles rather than specific aspects, such as details about drugs and their particular doses. We should not, in providing details, neglect to emphasise the extraordinary complexity of the treatment of these patients. Our experience shows that even very wise and experienced physicians will feel challenged. A remarkable amount of patience and handholding is essential. Working closely with families, we provide home care even for the most seriously affected patients. ${ }^{17}$
This approach is sensitive to the cost of care as well as the needs of $\frac{\text { W }}{\square}$ the patients and their families.

\section{References} 1 Newrick PG, Langton-Hewer R. Motor neurone disease: Can we do better? A study of 42 गु
patients. Br Med f 1984;289:539-42.

2 Smith RA, Norris FH. Symptomatic care of patients with amyotrophic lateral sclerosis. JAMA 1975;234:715-7.

3 Norris FH. Adult spinal motor neuron disease. In: Vinken PJ, Bruyn GW, eds. Handbook of clinical neurologv. Vol 22. Pt 2. Amsterdam, Oxford: North-Holland Publishing Co, 1975:1-56. ${ }^{\circ}$ 4 Mills CP. Dysphagia in pharyngeal paralysis treated by cricopharyngeal sphincterotomy. Lancet 1973;i:455-7.

5 Lebo CP, U KS, Norris FH. Cricopharyngeal myotomy in amyotrophic lateral sclerosis. Laryngoscope 1976;86:862-8.

$6 \mathrm{U} \mathrm{KS}$, Norris FH, Denys EH, Lebo CP. Surgery in patients with amyotrophic lateral sclerosis. Experience with cricopharyngeal myotomy in 100 cases. Proceedings of XI World Congress of $\frac{\mathrm{S}}{\mathrm{O}}$ Neurology. Amsterdam: Excerpta Medica, 1977:45. (International Congress Series No 427. $\mathrm{d}$
Abstract 134.)

7 Fallat RJ, Jewitt B, Bass M, Kamm B, Norris FH. Spirometry in amyotrophic lateral sclerosis. ص్ Arch Neurol 1979;36:74-80.

8 Gauderer MWL, Ponsky JL, Izant RJ. Gastrostomy without laparotomy: a percutaneous $\vec{\circ}$ endoscopic technique. F Pediatr Surg 1980;15:872-5

9 Russell TR, Brotman M, Norris FH. Percutaneous gastrostomy: a new simplified and cost- $\overrightarrow{-}$ effective technique. Am $\mathcal{F}$ Surg 1984;148:132-7.

10 Rivera VM, Breitbach WB, Swanke L. Dantrolene in amyotrophic lateral sclerosis. FAMA 1975;233:862-3.

11 Sydenham T. Epistolae responsoriae I (1669). In: Latham RG, ed. The works of Thomas Sydenham

MD. Vol 2. London: Sydenham Society, 1948 .
12 Mulder DW, Lambert EH, Eaton LM. Myasthenic syndrome in patients with amyotrophic lateral sclerosis. Neurology 1959;9:627-31.

13 Denys EH, Norris FH. Amyotrophic lateral sclerosis: impairment of neuromuscular transmission. क Arch Neurol 1979;36:202-5.

14 Norris FH, U KS, Denys EH, Archibald KC, Lebo C. Amyotrophic lateral sclerosis. Mayo Clin

Proc 1978;53:544.
15 Lenman JAR. A clinical and experimental study of the effects of exercise on motor weakness in neurological disease. I Neurol Neurosurg Psychiatry 1959;22:182-4.

16 Moxley RT. The role of exercise. In: Mulder DM, ed. The diagnosis and treatment of amyotrophic lateral sclerosis. Boston: Houghton-Mifflin, 1980:195-214.

17 Norris FH, Smith RA, Denys EH, et al. Home care of the paralyzed respirator patient. In:

Kutscher LG, ed. Coping with progressive disability. Springfield: Thomas (in press).
18 Janiszewski DW, Caroscio JT, Wisham LH. Amyotrophic lateral sclerosis: a comprehensive rehabilitation approach. Arch Phys Med Rehabil 1983;64:304-7.

19 Perry AR, Gawel M, Rose FC. Communication aids in patients with motor neurone disease. $\mathrm{Br} \underset{\bullet}{\vec{O}}$ Med F 1981;282:1690-3.

20 Denys EH. Living without speech. In: Mulder DW, ed. The diagnosis and treatment of amyotrophic lateral sclerosis. Boston: Houghton-Mifflin, 1980:235-40.

21 Bradley WG. Respirator support in amyotrophic lateral sclerosis. Ann Neurol 1982;12:466.

22 Bradley WG. Doctor-patient relationships in ALS and muscular dystrophy. In: Kutscher LG, ed.

Coping with progressive disability. Springfield: Thomas (in press).
23 Sivak ED, Gipson WT, Hanson MR. Long-term management of respiratory failure in amyotrophic lateral sclerosis. Ann Neurol 1982;12:18-23.

24 Sivak ED, Cordasco EM, Gipson WT. Pulmonary mechanical ventilation at home: a reasonable $\frac{2}{\mathrm{O}}$ and less expensive alternative. Respiratory Care 1983;28:42-9.

25 Levinsky NG. The doctor's master. N Engl f Med 1984;311:1573-5.

5 Levinky NG. The doctor's master. N Englf Med 1984;311.1573-5. $\overrightarrow{\vec{B}}$ Udaka F, Yamao S, Nagata H, Nakamura S, Kameyama M. Pathologic laughing and crying $\frac{\mathrm{O}}{3}$
treated with levodopa. Arch Neurol 1984;41:1095-6. Accepted 11 April 1985)
What is the prognosis for a patient who has had a Charnley hip prosthesis for 10 years? What sort of exercises and activities would be of benefit for him?

The prognosis of a Charnley total hip replacement is influenced by three major factors-namely, infection, wear, and loosening. In a patient whose implant has been in situ for 10 years infection is unlikely to occur. Normally, infection occurs soon after operation, or within the first two to three years. It is uncertain whether this infection arises from a nidus trapped at the time of the operation or haematogenous infection at a later date. Unfortunately, it is still possible for this complication to occur 10 years after the operation, in which case revision would be indicated. The problem of wear of the plastic component depends on the age and activity of the patient. A young, active patient is more likely to wear out his prosthesis than a sedentary, old one. Drastic wear is unlikely in patients whose prosthesis was inserted when they were over 60. Loosening may occur in all patients. Sometimes it occurs if the component has been incompletely surrounded by cement, thus permitting movement of the implant, which will result in its breaking away from the bone. Bone resorption or softening will also produce similar loosening, as may implant fracture. Although the original "flat-back" femoral components occasionally fracture because of metal fatigue, this is rare with the current implants. In summary, a patient who has had his Charnley prosthesis for 10 years, provided that it was put in when he was over 60 , is unlikely to have any complications. No specific exercises are necessary. Activities need not be curtailed if the patient is over 60 , but under this age the patient should be advised to ride rather than walk, and not to engage in vigorous sport (tennis, squash, etc). Fortunately, the occasional round of golf seems to benefit both patient and hip joint.-C D R LIGHTOWLER, consultant orthopaedic surgeon, London.
What is the effect of ingesting supplementary fluoride by adults living in an area where the fluoride content of the mains water supply is low? In particular, does it delay the process of senile osteoporosis?

The effects of long term ingestion of fluoride are critically dependent ono dosage. Fluoride has been assessed in several studies on the treatment of osteoporosis but the doses used are much greater (average $60 \mathrm{mg}$ a day) than those normally recommended for supplementation in people using a non- $\rightarrow$ fluoridated water supply (usually $2 \cdot 2 \mathrm{mg}$ a day). In the presence of osteoporosis the administration of doses sufficient to maintain a serum $\mathrm{N}$ fluoride concentration between 5 and $10 \mu \mathrm{M}$ does appear to increase trabecular bone volume, reduce pain, and lower the rate of spontaneous $\omega$ fracture. Lower doses are ineffective and higher doses produce abnormalo bone. There is a high incidence of side effects consisting of synovitis in theo large joints of the legs, plantar fasciitis, and gastrointestinal upset. I know of

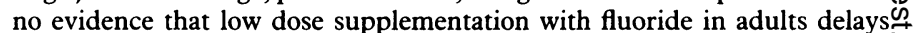
senile osteoporosis. Such practice must be extremely rare and to provide 0 such evidence would require a massive prospective epidemiological study. $\overline{0}$ Subjects living in areas with fluoridated water have greater bone density than $\mathbb{\Phi}$ comparable subjects living in areas where fluoride levels are low. ${ }^{1}$ In animals, $\frac{\rho}{\mathrm{D}}$ however, low dose fluoride was ineffective in preventing either the bone loss $\varrho$ induced by a low calcium diet or glucocorticoid induced osteopenia. On the other hand, osteopenia due to disuse seen in tail vertebrae immobilised by neural resection did appear to be reduced or prevented by fluorideo supplementation. ${ }^{2}$ - CLIVE ROBERTS, consultant physician, Bristol.

1 Bernstein DS, Sadowsky, N, Hegsted DM, Guri CD, Stare FJ. Prevalence of osteoporosis in high and low fluoride areas in North Dakota. $\mathcal{J} A M A$ 1966;198:499-504.

2 Bikle DD. Fluoride treatment of osteoporosis: a new look at an old drug. Ann Intern Med 1983;98:1013-5. 